\title{
Temptation shapes dishonesty and can alter working memory
}

\author{
Andrea Pittarello ${ }^{1}$. Marcella Fratescu ${ }^{2} \cdot$ Sebastiaan Mathôt ${ }^{2}$
}

Accepted: 26 September 2021

(c) The Author(s) 2021

\begin{abstract}
This study shows that participants tend to remember an ambiguous, directional cue as biased towards stimuli associated with a high reward that can be attained dishonestly. Participants saw eight digits presented in a circular arrangement. On some trials, they were asked to report the digit ("Target Digit") indicated by a jittery cue that was slightly biased in the direction of another digit ("Second Cued Digit"), which was either higher or lower than the Target Digit. Participants were paid based on the reported digit (higher digits meant higher pay) and not based on the accuracy of their report. In this setting, they could make self-serving mistakes by dishonestly reporting the Second Cued Digit when it was higher than the Target Digit. Replicating prior work, participants frequently made such self-serving mistakes. On other trials, after the digits disappeared, participants were asked to reproduce the direction of the jittery cue, without receiving any pay. Results showed that that participants' reports of the cue were more biased toward high-rewarding digits than low-rewarding digits. This research provides preliminary evidence of a link between attention, dishonesty, and memory, offering an important constraint for theories in behavioral ethics.
\end{abstract}

Keywords Ethical blind spots $\cdot$ Morality $\cdot$ Memory $\cdot$ Temptation $\cdot$ Attention

\section{Introduction}

Everyday life presents many opportunities to profit from dishonesty, from academic cheating to stealing and fraud (Mazar \& Ariely, 2006). Although people condemn these behaviors and think of themselves as honest, they frequently bend the rules and lie (Bazerman \& Tenbrunsel, 2011). According to bounded-ethicality theory (Chugh et al., 2005) dishonesty often results from ethical blind spots: people's tendency to pay attention to tempting (but dishonest) information at the expense of non-tempting (but honest) information (Bazerman \& Tenbrunsel, 2011; Pittarello et al., 2015, for a similar definition). For instance, a CEO compiling the end-of-the-year report realizes that the returns do not look as good as he or she wished for. However, by adding some expenses that should be left out because non-deductible,

Sebastiaan Mathôt

s.mathot@rug.nl

1 Psychology Department, Virginia Polytechnic Institute and State University, 890 Drillfield Dr. 24061, Blacksburg, VA, USA

2 Psychology Department, University of Groningen, Grote Kruisstraat 2/1 9712 TS, Groningen, the Netherlands the CEO could reduce the taxable income to pay less taxes. In this setting, these 'tempting' expenses could capture the attention of the CEO creating a blind spot and a subsequent irregular report.

Despite the converging evidence that temptation makes people perceive reality in a self-serving way (Pittarello et al., 2019; Pittarello et al., 2015), it remains unknown if temptation also makes people remember reality as more rewarding than it was. This is the question addressed presently. This research links evidence that dishonest yet rewarding information captures attention with work showing that workingmemory is also biased towards rewarding stimuli (Gong \& $\mathrm{Li}, 2014)$. Specifically, it proposes that people's memory of a cue will be more biased toward stimuli associated with a high as compared to a low reward, even in situations where these stimuli no longer yield any payoff. In doing so, this works links for the first-time dishonesty, attention, and memory, to further the understanding of the low-level cognitive processes associated with morality. 


\section{Attention and Moral Behavior}

A recent line of research (Fiedler \& Glöckner, 2015; Hochman et al., 2016; Leib et al., 2019; Pittarello et al., $2015,2016,2019)$ measured eye movements to investigate what causes ethical blind spots and when they emerge. For instance, Pittarello et al. (2015) asked participants to report the outcome of a die roll appearing closest to a fixation cue, with higher reports meaning higher payoffs (irrespective of participants' accuracy). On some trials, the die second closest to the fixation cross was "tempting" in the sense that it was higher (and thus more profitable) than the target die. On these trials, participants made self-serving mistakes (an implicit form of dishonesty) by reporting the tempting die roll instead of the correct one. Crucially, tempting dice captured visual attention and created ethical blind spots: Participants fixated on these dice longer at the expense of fixations on the correct dice, and the strength of this effect in turn predicted the extent of their dishonesty.

In another recent study, Pittarello et al. (2019) used a variation of this experimental paradigm, in which they presented participants with a series of digits on a screen and asked them to report the digit indicated by a cue. There was some ambiguity in the orientation of the cue, such that at times it was slightly pointed toward another digit, which was either higher or lower than the correct digit. Participants were paid more for reporting higher digits (irrespective of whether they answered correctly) and were not penalized for making mistakes. Conceptually replicating prior work, participants made self-serving mistakes by reporting higher digits. Furthermore, tempting digits captured participants' gaze relatively quickly (for converging evidence, Hochman et al., 2016), suggesting that ethical blind spots and the resulting self-serving mistakes (at least in these settings, Shalvi et al., 2012) occur rapidly and without much deliberation. Taken together, this research clearly shows that dishonest stimuli associated with high rewards capture attention, which in turn shapes dishonesty.

\section{Rewards and Memory}

While novel in the field of morality, the effect of rewards on attention is well-established in the literature on visual attention (Failing \& Theeuwes, 2018). The general idea is that stimuli associated with rewards create an attentional template (Chelazzi et al., 1998; Reynolds et al., 1999) that increases their accessibility much like visual saliency does (Theeuwes, 1991, 1992, 2010). Importantly, such accessibility persists over time even when the same stimuli are no longer associated with rewards. For instance, Della and
Chelazzi (2009) asked participants to engage in a shapematching task in which they were rewarded for their correct responses. During the first part of the experiment, certain target and distractor shapes were associated with either a high or a low reward. In the second part of the experiment, participants performed the same task but received no rewards. The main finding was that performance in the second part of the task was affected by previously received rewards: Stimuli that were associated with high rewards acted as powerful distractors (and affected response accuracy) even when they were no longer associated with rewards, and thus, were irrelevant (for a similar conclusion, Anderson et al., 2011; Theeuwes \& Belopolsky, 2012).

Clearly, attention is biased by previously rewarded information (Failing \& Theeuwes, 2018), even when such rewards are obtained dishonestly (Pittarello et al., 2019). But is working memory also biased by reward? This paper tests whether participants' memory is biased toward prior rewards that could be achieved dishonestly. The prediction is based on prior research showing a connection between visual attention and working memory (Awh et al., 2006; Frătescu et al., 2019; Gazzaley \& Nobre, 2012; Zanto et al., 2011). The main idea is that "attention serves as a gatekeeper for memory" (Lange et al., 2012, p. 1179), meaning that an attended stimulus leaves a trace in memory that directs future tasks (Gong \& Li, 2014). For instance, Van der Stigchel et al. (2007) asked participants to remember the location of a target stimulus. On some trials, an irrelevant sudden distractor was presented (at a different location) shortly after the target stimulus. On other trials, no distractor was displayed. Results showed that participants remembered the target as biased towards the location of the distractor, especially when the distractor appeared near the target stimulus, indicating that working memory affected attention. Finally, and crucial to this research question, reward also improves the memory representations of stimuli associated with rewards (Gong \& $\mathrm{Li}, 2014)$.

To sum, previous research has shown that attention is biased towards rewarded stimuli, and that spatial workingmemory representations are biased towards attended stimuli. Therefore, this paper asked whether, in a task where participants frequently make self-serving mistakes by (mis) reporting rewarded stimuli, participants' memory is biased towards rewarded stimuli. This is possibly the first work that combines research on dishonesty, attention, and memory.

\section{The Current Study}

A modified version of the task previously used in Pittarello et al. (2019) was devised. Participants were presented with a series of digits (from 1 to 8 ) arranged in a circle and asked 
to report the digit indicated by an ambiguous cue displayed in the center of the screen (i.e., the Target Digit). Participants were paid based on the reported digit (irrespective of whether the report was correct), with higher reports corresponding to higher rewards. On some trials, the digit second closest to the cue, which we will refer to as the Second Cued Digit, was higher than the target (i.e., tempting), and on other trials it was lower (i.e., non-tempting). In this setup, participants who wish to maximize their gains can make 'self-serving mistakes' and report higher digits instead of the correct ones, in line with prior work (e.g., Leib et al., 2019; Pittarello et al., 2019; 2015). Past research using the same setup (Pittarello et al., 2019) included a control condition in which participants were paid only if they correctly reported the Target Digit. In this condition, participants report the Target Digit significantly more often than in an experimental treatment in which they are solely paid per report (and not per accuracy). This shows that they understand the instructions and the task and that their mistakes are systematic, meaning that they are not simply reporting higher digits. Thus, the present design does not include a control condition (for an identical result with a different setup see Pittarello et al., 2015)

To test whether working memory is biased towards tempting stimuli, the study included trials in which participants first saw the digits, and then - after the digits had disappeared - rotated the cue (using the mouse scroll) to the position in which it originally appeared; in other words, participants performed a basic delayed-estimation task as is often used in visual-working-memory research (e.g., Wilken \& Ma, 2004; Zhou et al., 2021). Participants did not receive any pay on these trials, meaning that the value of the digits was irrelevant for this task. Importantly, when the digits were presented and encoded into working memory, participants did not yet know whether they would be asked to report the cued digit - and hence earn money - or to reproduce the direction of the cue. Because prior research showed that tempting and dishonest information captures attention and that attention affects working memory, it is plausible that in this setting temptation would also affect the recollection of the cue, even though participants had no direct incentive to bias their recollection of the cue.

\section{Methods}

Participants Sixty participants took part in the study. Demographic information was collected for fifty participants $(80 \%$ Females, $\mathrm{M}_{\text {age }}=19.74$ years, $\mathrm{SD}_{\text {age }}=2.61$ years). Ten participants were removed due to a programming error, and 19 for incorrectly answering the manipulation check at the end of the procedure. Thus, the final sample consisted of 31 participants, in line with that of Pittarello et al. (2015;
2019) who used a similar methodology. All participants had normal or corrected vision and earned an average of $€ 2.16$ ( \$2.41). The study lasted \pm 20 min, was approved by the local ethics committee and all participants read and signed a consent form before participation.

Materials Participants saw 8 randomly presented digits (from 1 to 8 ) arranged on the circumference of an imaginary circle. One of these (the Target Digit) was indicated by a central, ambiguous cue. After 2,500 ms, the jittery cue disappeared, and participants were prompted to answer. Within-subjects, two types of trials were manipulated: On the "Report Digit" trials $(75 \% ; N=7,221)$, participants were paid according to the digit they reported, with higher digits corresponding higher payoffs (i.e., ' 1 ' $=€ 0.01$, ' 2 ' $=€ 0.02$, ' 3 ' $=€ 0.03,{ }^{\prime} 4$ ' $=€ 0.04,{ }^{\prime} 5$ ' $=€ 0.05, ' 6$ ' $=€ 0.06,{ }^{\prime} 7$ ' $=€ 0.07$, and ' 8 ' $=€ 0.08)$. On the "Report Rotation" trials $(25 \%$; $N=2,409)$ participants were asked to rotate the cue - using the mouse scroll - to where it corresponded before it disappeared. Thus, the two types of trials were similar, with the only difference being that after seeing the eight digits on the screen, on the "Report Digit" trials, participants had to report the digit that they thought was indicated by the cue (and were rewarded accordingly), whereas on the "Report Rotation" trials, they were asked to rotate the cue to how they thought it was its previously rotated without receiving any monetary payoff. For both trial types, the ambiguity of the jittery cue was manipulated such that it pointed slightly $(10 \%)$ or moderately $(40 \%)$ towards a second digit (i.e., "Second-Cued Digit") (see Fig. 1 for a schematic representation of the procedure). To incentivize reports, the value of the Second-Cued Digit was either higher than the Target Digit (i.e., tempting on the "Report Digit" trials), or lower than the Target Digit (i.e., non-tempting on the "Report Digit" trials). Participants who wanted to dishonestly earn more money could report the Second-Cued Digit instead of the correct Target Digit more often when it was tempting thus making self-serving mistakes - than when it was not tempting - thus avoiding self-hurting mistakes.

The complete experimental design included three withinsubject factors: Trial Type ("Report Digit" vs. "Report Rotation"), Cue Ambiguity ("low" vs. "moderate"), and Second-Cued Value ("Tempting" vs. "Non-Tempting"). Each participant completed 16 practice trials followed by 240 "Report Digit" and 80 "Report Rotation" trials. At the end of the experiment, participants answered the following manipulation check: "Please, pay attention! We want to make sure you understood how you earned money in this task. You received:". Participants chose between: "More money for a correct than an incorrect answer (regardless of whether you reported a high or low digit)", or "More money for reporting a high than a low digit (regardless of whether your answer was correct)". Finally, for exploratory purposes, 
Fig. 1 Schematic representation of the procedure. In this example, the Target Digit is ' 2 ' and the Second-Cued Value a ' 6 '. The jittery cue points moderately toward the tempting Second-Cued Value. In the Report Digit trials, participants reported the digit using the keyboard. In the Report Rotation trials, they reported the position of the cue using the mouse scrol
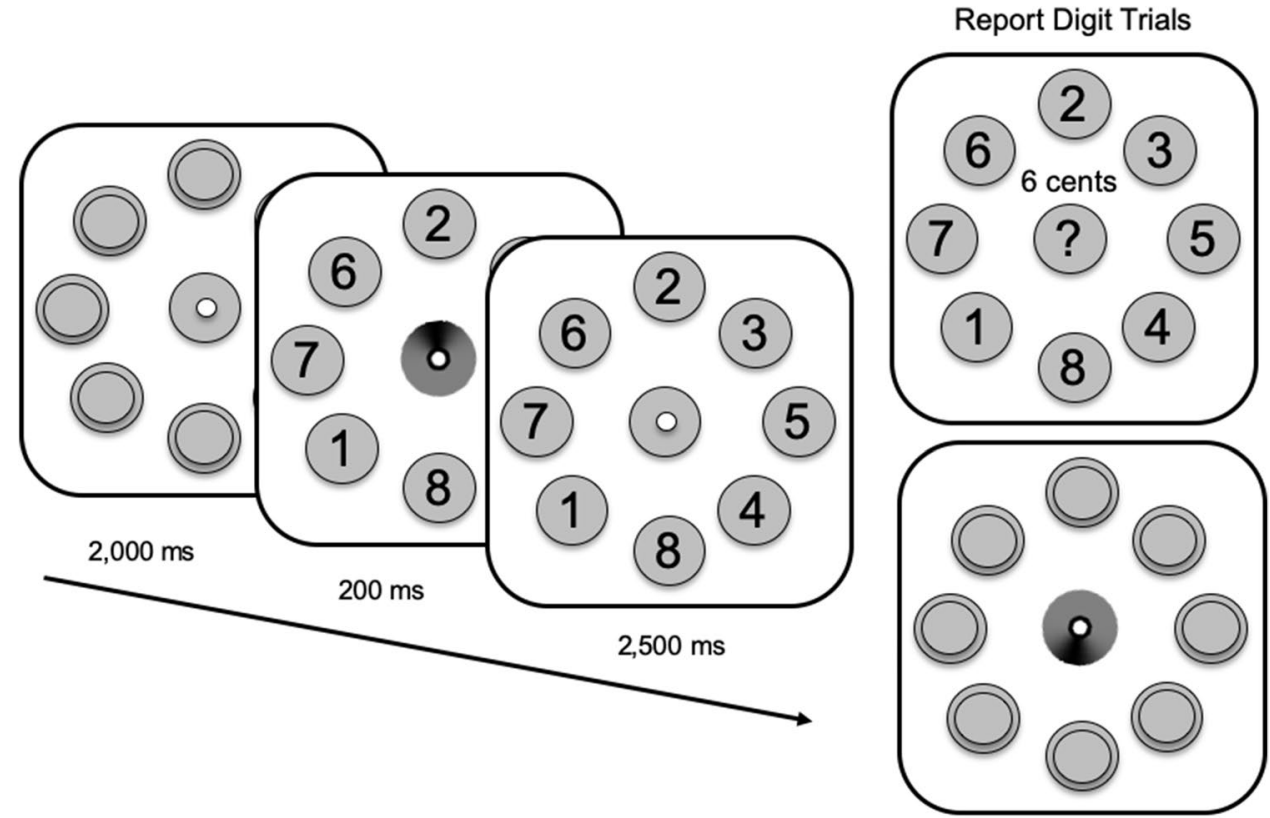

Report Rotation Trials they were asked the following question "On a scale from 1 (not at all) to 7 (very much), to what extent do you think you accurately adjusted the cue?". Since this question did not significantly correlate with any of the other variables of interest, it will not be discussed further.

\section{Results}

In the sections below, we report separate analyses for the different types of trial. We first report the report digit trials (where participants can make self-serving mistakes and profit from dishonesty). Next, we discuss the rotation trials, in which we test whether participants (mis)remember the cue a pointing towards a high vs. low digit. Regarding the report digit trials, we refer to Table 1 below which displays participants' reports in percentages (i.e., Target, SecondCued, Highest Digit displayed, and Other Digit) depending on whether the Second-Cued Digit was higher or lower than the Target Digit (i.e., temptation). Overall, we report two key findings: First, we find evidence of self-serving mistakes. Second, we show that participants tend to remember the cue as pointing more toward a tempting than a non-tempting digit. Details on the analyses are reported below.

Report Digit Trials A series of Wilcoxon signed-rank Bayesian paired-samples t-tests (using the default parameters of JASP 0.13.1) showed evidence $(B F 10=211.66)$ for a difference in participants' overall likelihood to report the Target Digit (38.33\%) vs. the Second-Cued Digit (19.80\%); this simply reflects that participants, on average but imperfectly, followed the task instruction to report the Target Digit. The interest was in participants' mistakes, meaning situations in which they incorrectly reported the Second-Cued Digit instead of the Target Digit (i.e., the correct response). In line with prior work, participants' mistakes were systematic and self-serving. Specifically, participants were $\sim 5$ times more likely to report the Second-Cued Digit when it was tempting as compared to when it was not tempting $(\mathrm{BF} 10=11,769.68)$. This finding conceptually replicates prior work (Leib et al., 2019; Pittarello et al., 2015; 2019). Conversely, participants were less likely to report the Target Digit when the Second-Cued Digit was tempting as compared to when it was not tempting $(\mathrm{BF} 10=3587.86)$ (see Table 1).

A Bayesian repeated measures ANOVA with ambiguity, Second-Cued Value and their interaction as predictors and likelihood to report the Second-Cued Digit as dependent variable was conducted. When comparing the model with the interaction to the best model with Second-Cued

Table 1 Reported outcome (in \%) as function of temptation. In total, there were $N=7,221$ trials

\begin{tabular}{llllll}
\hline & & \multicolumn{3}{c}{ Digit Reported (\%) } & \\
\cline { 3 - 5 } & & Target & Second-Cued & Highest & Other \\
\hline $\begin{array}{c}\text { Second- } \\
\text { Cued } \\
\text { Value }\end{array}$ & Tempting & $24.65 \%$ & $33.51 \%$ & $28.01 \%$ & $13.83 \%$ \\
& $\begin{array}{c}\text { Non-Tempt- } \\
\text { ing }\end{array}$ & $52.01 \%$ & $6.09 \%$ & $28.64 \%$ & $13.26 \%$ \\
& & & & & \\
\hline
\end{tabular}


Value only it was found evidence against the interaction $(\mathrm{BF} 10=0.27)$. As can be seen in Table 1 , some participants ("brazen liars") reported the highest digit (' 8 ') on almost every trial clearly showing that they were not sensitive to the temptation, but simply maximized their profit (Pittarello et al., 2019; Weisel \& Shalvi, 2015 for a similar pattern). For robustness purposes, the same repeated measures Bayesian ANOVA was conducted after removing these participants (whose average reported digit was $\geq 7.5$ ) and found evidence against the interaction $(\mathrm{BF} 10=0.31)$.

In sum, participants made systematic mistakes: When incorrectly reporting the Second-Cued Digit, they were $\sim 5$ more likely to do so when it was higher vs. lower than the Target Digit.

Report Rotation Trials The Cue Report Error was normalized, such that an error of 0 corresponds to a perfect reproduction of the cue orientation, whereas positive errors correspond to errors in the direction of the Second-Cued Digit and negative errors correspond to errors in the direction of the Target (the cue always pointed at an intermediate location, but closer to the Target than to the Second Cued Digit). A distribution of Cue Report Errors is shown in Fig. 2a, b.

Next, a so-called "mixture model" to the Cue Report Errors (using the Biased Memory Toolbox; Zhou et al., 2021) was applied, a technique that is often used in visualworking-memory research (Zhang \& Luck, 2008) to model responses in delayed-estimation tasks. The mixture model used here consists of a weighted mix of a Von Mises distribution (a normal distribution for rotational values) and a uniform distribution. The relative weighting of these distributions is determined by the Guess Rate parameter, such that a Guess Rate of 1 corresponds to a pure uniform distribution (random responses) and a Guess Rate of 0 corresponds to a pure Von Mises distribution (non-random responses). The width of the Von Mises distribution is determined by the Precision parameter, such that higher Precision values correspond to a narrower distribution (i.e., less variability in the responses). Finally, the shift of the Von Mises distribution along the $\mathrm{x}$-axis is determined by the Bias parameter, such that positive Bias values correspond (in this experiment) to a bias towards the Second Cued Digit. The main prediction concerns the Bias parameter, which was expected to be higher on tempting trials, where the Second Cued Digit is higher than the Target, as compared to non-tempting trials.

This model was fitted separately for each combination of participant and Second Cued Value (Tempting, Non-Tempting; Fig. 2c). Next, a Bayesian paired-samples t-tests was used to test whether the model parameters differed between Tempting and Non-Tempting trials (using the default parameters of JASP 0.13.1). There was no no evidence for a difference in the Precision $(B F 10=0.56)$ parameter between Tempting and Non-Tempting trials, and even some evidence against a difference in the Guess Rate parameter $(\mathrm{BF} 10=0.20)$. However, as predicted, there was "strong evidence" for a difference in the Bias parameter, which was higher on Tempting trials than on Non-Tempting trials $(\mathrm{BF} 10=18.76)$; that is, participants remembered the cue as pointing more towards the Second Cued Digit on those trials where the Second Cued Digit was tempting, as compared to when it was not tempting.

\section{Discussion}

When facing ethical dilemmas, temptation biases how people see and potentially remember reality. This paper suggests that participants' memory is biased toward high-reward stimuli, even at moments when such stimuli do not yield any payoff. In this experiment, participants saw a series of digits associated with different payoffs (higher digits meant higher pay) preceded by an ambiguous cue. On some trials, which served to associate the different digits with different levels of reward, participants were asked to report the cued digit. The cue was slightly tilted in the direction of another digit that could be either higher (i.e., tempting) or lower (i.e., non-tempting) than the cued digit. Results successfully replicated prior work (e.g., Leib et al., 2019; Pittarello et al., 2015; 2019), and found that participants made self-serving mistakes (an implicit form of dishonesty) by frequently reporting higher digits instead of cued and correct digits; participants rarely made self-hurting mistakes, showing that responses are systematic and that participants understand the reward scheme.

On other trials, which served to measure the effect of reward on visual working memory, after the digits and the cue disappeared, participants were prompted to rotate the cue back to how it was previously displayed. Importantly, when the digits were presented and encoded into working memory, participants did not yet know whether they would be asked to report a digit (and thus be rewarded), or to reproduce the orientation of the cue (with no chance of reward). On these trials, there was strong evidence of a bias towards tempting digits in the reported cue orientations. Phrased differently, participants remembered the cue as pointing more towards highly rewarded digits than it really was. ${ }^{1}$

\footnotetext{
${ }^{1}$ It is important to note that participants - except for some brazen liars - reported honestly (i.e., the Target Digit) on more than $20 \%$ of the trials even the Second-Cued Digit was tempting, suggesting that they are not merely reporting the highest digits. Furthermore, prior research using the same procedure (Pittarello et al., 2019) showed that participants are not affected by high digits when rewarded per accuracy. For these reasons, it is unlikely that participants simply rotated the cue towards high digits, especially when there were no monetary incentives to do so. This notwithstanding, future work could corroborate these findings for instance by including a condition in which reward value and digit value are not confounded.
} 
a) Second Cued > Target
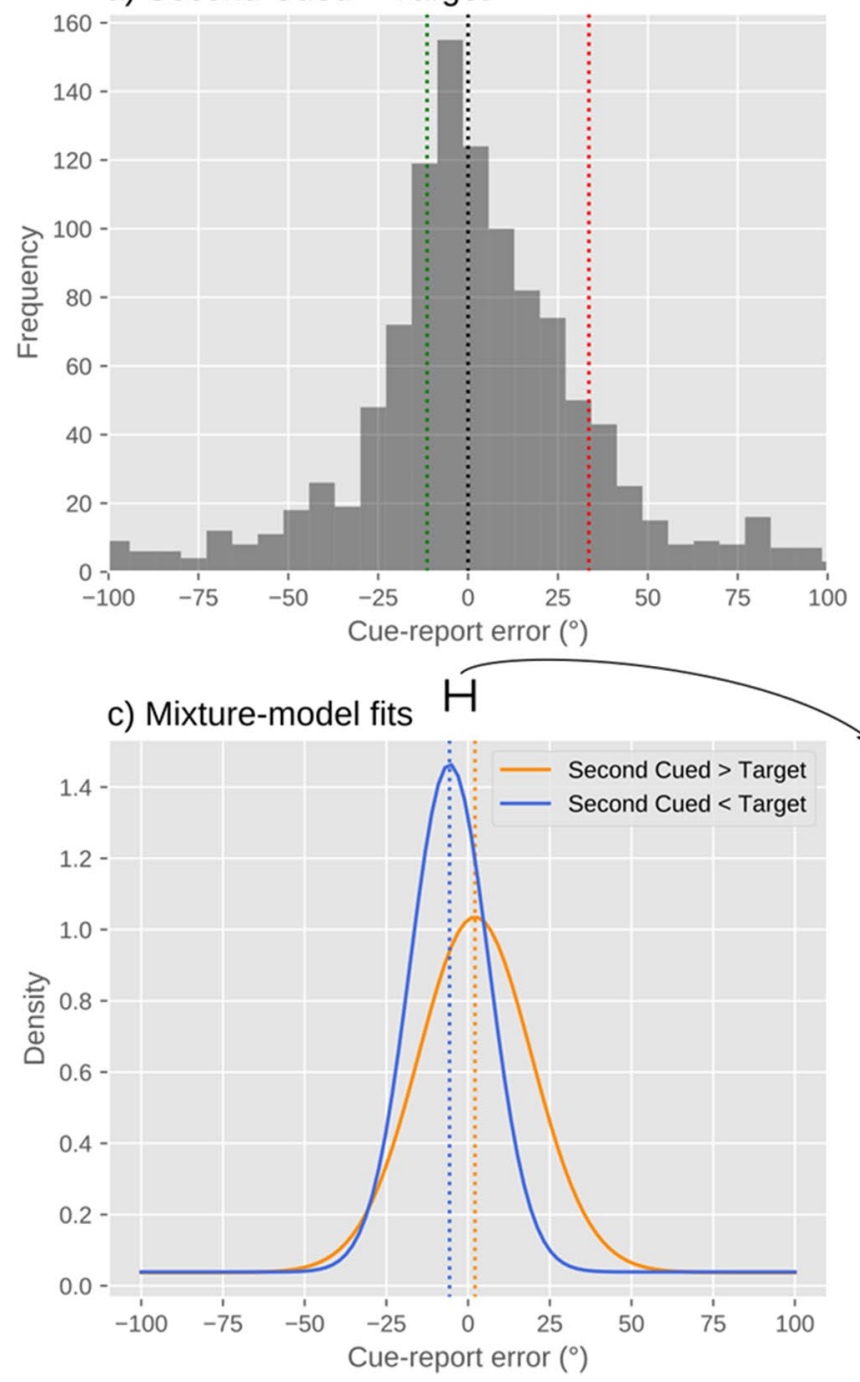

Fig. 2 The distribution of Cue Report Errors for "tempting" trials where the Second Cued digit was higher than the Target (a) and "non-tempting trials" where the Second Cued digit was lower than the Target (b); here, an error of 0 corresponds to a perfect reproduction of the cue orientation, and positive errors correspond to an error in the direction of the Second Cued Digit. The red dotted line corresponds to the mean direction of the Second Cued Digit (relative to the cue). The green dotted line corresponds to the mean direction of the target (relative to the cue). (c) The results of a mixture model

These results are consistent with studies showing that reward affects visual attention (reviewed in Failing \& Theeuwes, 2018), and that visual attention in turn affects visual working memory (e.g., Van der Stigchel et al., 2007). The novelty of the present study lies in linking these findings to research on morality, specifically on the cognitive processes associated with ethical blind spots and self-serving mistakes. This area of research is relatively recent and understudied. For instance, As Leib and colleagues reported (2019), "unlike outright lies that are by definition conscious, b) Second Cued $<$ Target

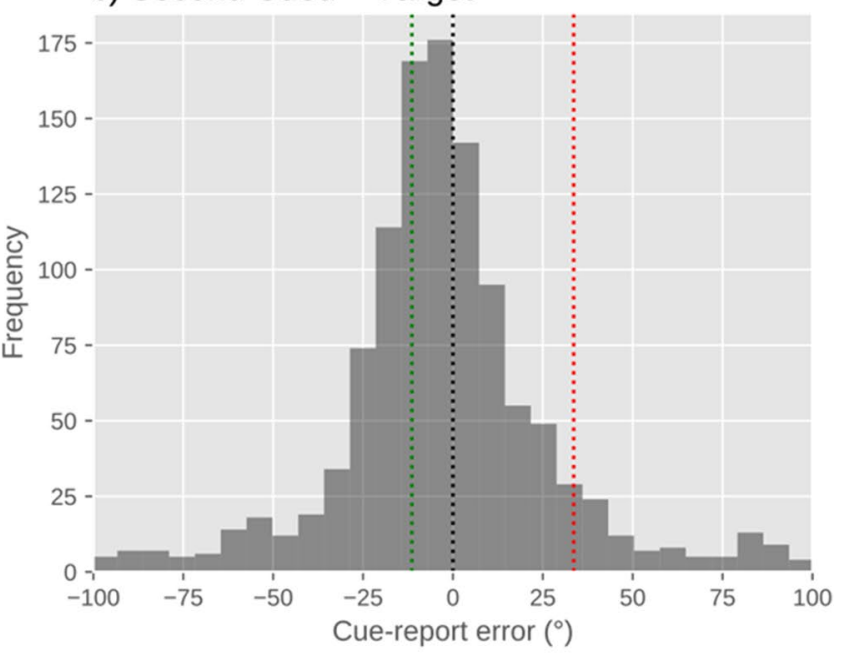

\section{d) Memory bias for individual participants}

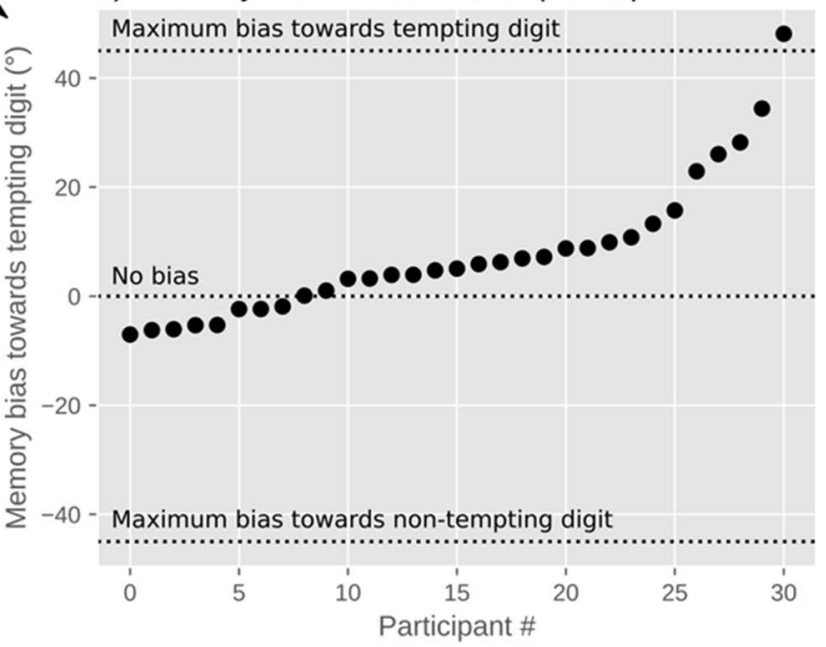

applied to the tempting (orange) and non-tempting (blue) trials. The distributions shown here are based on the mean parameters across participants. For details about the model, see the main text. (d) The difference in the Bias parameter between Tempting and Non-Tempting trials ("temptation bias"; y-axis) for individual participants. A positive bias of $45^{\circ}$ is a maximum bias in the sense that, on average, the cue was remembered as pointing towards the Second Cued Digit when it was higher than the Target, and as pointing towards the Target otherwise

whether self-serving mistakes are conscious or not remains an open question" (p. 10). Accordingly, Hochman and colleagues (2016) found that people exhibited physiological arousal when they lie, which suggests that they are aware of their wrongdoings (Mazar et al., 2008). However, at the same time they also found an attentional bias toward dishonest alternatives, in line with Pittarello et al. (2019). Thus, it is possible that self-serving mistakes involve both lowand high-level cognitive processes. While prior work has shown that blind spots affect visual perception, this work 
suggests that that they can also (at least to an extent) alter memory. This hints toward the possibility that dishonesty (at least in this context) might be associated with biases in working memory. For these reasons, the findings of these research can be situated and interpreted in the light of prior work on "bounded ethicality". To shed further insights into the automaticity of 'self-serving mistakes', future research could use simpler tasks or assess either eye movements or reactions times, measures that are more likely to reflect automatic reactions and fall outside people's volitional control (Theeuwes \& Belopolsky, 2012).

The connection between memory and morality has been recently investigated but yielded inconsistent results. Research has focused on episodic long-term memory rather than, as in this study, working memory. For instance, Kouchaki and Gino (2016) found that people are likely to forget their past misbehaviors. However, a recent paper failed to replicate this result (Stanley et al., 2018), suggesting that the link between episodic memory and dishonesty is unclear and deserves further attention. This work - though using different measures of dishonesty and focused on working rather than long-term memory - adds to this intriguing and unexplored area of research by cautiously suggesting that when cheating is possible, tempting, and dishonest information can make people remember reality in a self-serving way.

Finally, a pattern of brazen liars emerged - participants who disregarded instructions and simply maximized their payoff. This finding is consistent with prior work (Pittarello et al., 2019; Weisel \& Shalvi, 2015) and literature suggesting heterogeneity in dishonesty (Hilbig \& Thielmann, 2017). Although the focus of the present work was not on individual differences, such differences clearly do exist. Assessing individual differences such as Honesty/Humility (see Klein et al., 2020) can identify individuals who are more (or less) sensitive to temptation and incentives. This can help devising fine-grained interventions to fight dishonesty.

In summary, participants were instructed to report a target stimulus that was indicated by a central cue. It was found that participants tended to make self-serving mistakes by (mis)reporting non-target stimuli that were associated with a high reward. Crucially, this tendency to 'cheat' was accompanied by a bias in visual working memory, such that participants remembered the cue as being oriented more towards high-reward stimuli than it really was. To conclude, it is possible that biases in visual working memory may be a mechanism that underlies some forms of implicit dishonest behavior, such as self-serving mistakes.

Acknowledgements This research was supported by a collaboration grant awarded to Andrea Pittarello and Sebastiaan Mathôt by the University of Groningen.
Data Availability All data and experimental materials can be found at https://osf.io/npbmg/?view_only=85d7c92db3da4c2da5e5014e1 d8a7ca0.

\section{Declarations}

Conflict of Interest The authors have no relevant financial or non-financial interests to disclose.

Open Access This article is licensed under a Creative Commons Attribution 4.0 International License, which permits use, sharing, adaptation, distribution and reproduction in any medium or format, as long as you give appropriate credit to the original author(s) and the source, provide a link to the Creative Commons licence, and indicate if changes were made. The images or other third party material in this article are included in the article's Creative Commons licence, unless indicated otherwise in a credit line to the material. If material is not included in the article's Creative Commons licence and your intended use is not permitted by statutory regulation or exceeds the permitted use, you will need to obtain permission directly from the copyright holder. To view a copy of this licence, visit http://creativecommons.org/licenses/by/4.0/.

\section{References}

Anderson, B. A., Laurent, P. A., \& Yantis, S. (2011). Value-driven attentional capture. Proceedings of the National Academy of Science, 108(25), 10367-10371. https://doi.org/10.1073/pnas.11040 47108

Awh, E., Vogel, E. K., \& Oh, S. H. (2006). Interactions between attention and working memory. Neuroscience, 139(1), 201-208. https://doi.org/10.1016/j.neuroscience.2005.08.023

Bazerman, M. H., \& Tenbrunsel, A. E. (2011). Blind spots: Why we fail to do what's right and what to do about it. Princeton, NJ: Princeton University Press. https://doi.org/10.1515/9781400837991

Chelazzi, L., Duncan, J., Miller, E. K., \& Desimone, R. (1998). Responses of neurons in inferior temporal cortex during memory-guided visual search. Journal of Neurophysiology, 80(6), 2918-2940. https://doi.org/10.1152/jn.1998.80.6.2918

Chugh, D., Bazerman, M. H., \& Banaji, M. R. (2005). Bounded ethicality as a psychological barrier to recognizing conflicts of interest. In D. A. Moore, D. M. Cain, G. Loewenstein, \& M. H. Bazerman (Eds.), Conflicts of interest: Challenges and solutions in business, law, medicine, and public policy (pp. 74-95). Cambridge University Press.

Della, L. C., \& Chelazzi, L. (2009). Learning to attend and to ignore is a matter of gains and losses. Psychological Science, 20(6), 778784. https://doi.org/10.1111/j.1467-9280.2009.02360.x

Failing, M., \& Theeuwes, J. (2018). Selection history: How reward modulates selectivity of visual attention. Psychonomic Bulletin \& Review, 25(2), 514-538. https://doi.org/10.3758/ s13423-017-1380-y

Fiedler, S., \& Glöckner, A. (2015). Attention and moral behavior. Current Opinion in Psychology, 6, 139-144. https://doi.org/10.1016/j. copsyc.2015.08.008

Frătescu, M., Van Moorselaar, D., \& Mathôt, S. (2019). Can you have multiple attentional templates? Large-scale replications of Van Moorselaar, Theeuwes, and Olivers (2014) and Hollingworth and Beck (2016). Attention Perception \& Psychophysics, 81(8), 2700-2709. https://doi.org/10.3758/s13414-019-01791-8

Gazzaley, A., \& Nobre, A. C. (2012). Top-down modulation: Bridging selective attention and working memory. Trends in Cognitive Sciences, 16(2), 129-135. https://doi.org/10.1016/j.tics.2011.11.014 
Gong, M., \& Li, S. (2014). Learned reward association improves visual working memory. Journal of Experimental Psychology: Human Perception and Performance, 40(2), 841-856. https://doi.org/10. 1037/a0035131

Hilbig, B. E., \& Thielmann, I. (2017). Does everyone have a price? On the role of payoff magnitude for ethical decision making. Cognition, 163, 15-25. https://doi.org/10.1016/j.cognition.2017.02.011

Hochman, G., Glöckner, A., Fiedler, S., \& Ayal, S. (2016). "I can see it in your eyes": Biased processing and increased arousal in dishonest responses. Journal of Behavioral Decision Making, 29(2-3), 322-335. https://doi.org/10.1002/bdm.1932

JASP Team (2020). JASP (Version 0.14.1)[Computer software].

Klein, S. A., Thielmann, I., Hilbig, B. E., \& Heck, D. W. (2020). On the robustness of the association between Honesty-Humility and dishonest behavior for varying incentives. Journal of Research in Personality, 88, 104006. https://doi.org/10.1016/j.jrp.2020. 104006

Kouchaki, M., \& Gino, F. (2016). Memories of unethical actions become obfuscated over time. Proceedings of the National Academy of Sciences, 113(22), 6166-6171. https://doi.org/10.1073/ pnas. 1523586113

Lange, E. B., Starzynski, C., \& Engbert, R. (2012). Capture of the gaze does not capture the mind. Attention Perception \& Psychophysics, 74(6), 1168-1182. https://doi.org/10.3758/s13414-012-0318-8

Leib, M., Pittarello, A., Gordon-Hecker, T., Shalvi, S., \& Roskes, M. (2019). Loss framing increases self-serving mistakes (but does not alter attention). Journal of Experimental Social Psychology, 85, 103880. https://doi.org/10.1016/j.jesp.2019.103880

Mazar, N., \& Ariely, D. (2006). Dishonesty in everyday life and its policy implications. Journal of Public Policy \& Marketing, 25(1), 117-126. https://doi.org/10.1509/jppm.25.1.117

Pittarello, A., Frătescu, M., \& Mathôt, S. (2019). Visual saliency influences ethical blind spots and (dis)honesty. Psychonomic Bulletin \& Review, 26(5), 1-10. https://doi.org/10.3758/ s13423-019-01638-1

Pittarello, A., Leib, M., Gordon-Hecker, T., \& Shalvi, S. (2015). Justifications shape ethical blind spots. Psychological Science, 26(6), 794-804. https://doi.org/10.1177/0956797615571018

Pittarello, A., Motro, D., Rubaltelli, E., \& Pluchino, P. (2016). The relationship between attention allocation and cheating. Psychonomic Bulletin \& Review, 23(2), 609-616. https://doi.org/10.3758/ s13423-015-0935-z

Reynolds, J. H., Chelazzi, L., \& Desimone, R. (1999). Competitive mechanisms subserve attention in macaque areas V2 and V4. Journal of Neuroscience, 19(5), 1736-1753. https://doi.org/10. 1523/JNEUROSCI.19-05-01736.1999
Shalvi, S., Eldar, O., \& Bereby-Meyer, Y. (2012). Honesty requires time (and lack of justifications). Psychological Science, 23(10), 1264-1270. https://doi.org/10.1177/0956797612443835

Stanley, M. L., Yang, B. W., \& De Brigard, F. (2018). No evidence for unethical amnesia for imagined actions: A failed replication and extension. Memory \& Cognition, 46(5), 787-795. https://doi.org/ 10.3758/s13421-018-0803-y

Theeuwes, J. (1991). Exogenous and endogenous control of attention: The effect of visual onsets and offsets. Attention Perception \& Psychophysics, 49(1), 83-90. https://doi.org/10.3758/BF032 11619

Theeuwes, J. (1992). Perceptual selectivity for color and form. Perception \& Psychophysics, 51(6), 599-606. https://doi.org/10.3758/ BF03211656

Theeuwes, J. (2010). Top-down and bottom-up control of visual selection. Acta Psychologica, 135(2), 77-99. https://doi.org/10.1016/j. actpsy.2010.02.006

Theeuwes, J., \& Belopolsky, A. V. (2012). Reward grabs the eye: Oculomotor capture by rewarding stimuli. Vision Research, 74, 80-85. https://doi.org/10.1016/j.visres.2012.07.024

Van der Stigchel, S., Merten, H., Meeter, M., \& Theeuwes, J. (2007). The effects of a task-irrelevant visual event on spatial working memory. Psychonomic Bulletin \& Review, 14(6), 1066-1071. https://doi.org/10.3758/BF03193092

Weisel, O., \& Shalvi, S. (2015). The collaborative roots of corruption. Proceedings of the National Academy of Science, 112(34), 10651-10656. https://doi.org/10.1073/pnas.1423035112

Wilken, P., \& Ma, W. J. (2004). A detection theory account of change detection. Journal of Vision, 4(12), 11-11. https://doi.org/10. $1167 / 4.12 .11$

Zanto, T. P., Rubens, M. T., Thangavel, A., \& Gazzaley, A. (2011). Causal role of the prefrontal cortex in top-down modulation of visual processing and working memory. Nature Neuroscience, 14(5), 656-661. https://doi.org/10.1038/nn.2773

Zhang, W., \& Luck, S. J. (2008). Discrete fixed-resolution representations in visual working memory. Nature, 453(7192), 233-235. https://doi.org/10.1038/nature06860

Zhou, C., Loris, M., M., \& Mathôt, S. (2021). Categorical bias in visual working memory: The effect of memory load and retention interval (retrieved from https://osf.io/puq4v/).

Publisher's Note Springer Nature remains neutral with regard to jurisdictional claims in published maps and institutional affiliations. 